\title{
DETERMINANTES DE LA JEFATURA FEMENINA EN LOS HOGARES MEXICANOS: MODELOS LOGIT Y PROBIT (2008-2020)
}

\author{
DETERMINANTS OF FEMALE \\ HEADSHIP IN MEXICO: \\ A LOGIT AND PROBIT APPROACH (2008-2020)
}

\author{
Magnolia Miriam Sosa Castro \\ (iD) orcid.org/0000-0002-6597-5293 \\ Universidad Autónoma Metropolitana \\ México \\ msosac87@hotmail.com
}

\section{Jorge Eduardo Castro Olivares}

(iD) orcid.org/0000-0002-4623-8087

Universidad Autónoma Metropolitana

México

jorge_castro.o@hotmail.com

\begin{abstract}
Women's participation as head of household has increased in the last years. Women are in a disadvantageous position because of lower labor force participation rate, structural and direct violence, among others. Women's headship results in a higher poverty incidence in households. This article, analyzes the influence of age, education level, income and number of family members younger than eleven years old, on the probability of a household being led by a woman, using a probabilistic model with binary response and data from 2008-2020.
\end{abstract}

Keywords: Female household headship, Logit, México, Probit.

\section{Resumen}

La participación de mujeres como jefas del hogar en México ha aumentado en los últimos años. Las mujeres están en situaciones desventajosas por menor participación económica, violencia estructural y directa, entre otras causas. La jefatura femenina expone a dichos hogares a una gran vulnerabilidad respecto a la incidencia en pobreza. El presente trabajo, analiza descriptiva y econométricamente el impacto que tienen variables como la edad, el nivel de educación, ingreso e integrantes menores de once años, en la probabilidad de que la jefatura del hogar corresponda a una mujer, mediante un modelo probabilístico de respuesta binaria en el periodo 2008-2020.

Palabras clave: jefatura del hogar femenina, México, modelo Logit, modelo Probit. 


\section{Introducción}

De acuerdo con diversos estudios sociodemográficos, la jefatura femenina en hogares mexicanos ha crecido en las últimas décadas. Con base en el Consejo Nacional de Evaluación de la Política de Desarrollo Social (Coneval), la composición de jefaturas en 2008 fue de 33 mujeres por cada 100 hombres, mientras que en 2018 se incrementó a 40 jefas por cada 100 jefes (Coneval, 2020).

Las causas que determinan la jefatura femenina de un hogar son variadas y responden a diversos factores, ya sean socioeconómicos, culturales $\mathrm{y} / \mathrm{o}$ demográficos. Demográficamente, las mujeres tienen una mayor esperanza de vida que los hombres; como resultado, la proporción de jefas en el grupo de 65 años o más es de 63\% (Coneval, 2020).

En cuanto a los aspectos culturales, las nuevas pautas de nupcialidad, caracterizadas por un incremento de separaciones $y$ divorcios, se relacionan con la creciente probabilidad de formación de hogares monoparentales o ampliados encabezados por mujeres. En 2018, de los hogares con jefas $45 \%$ fueron nucleares y $48 \%$ ampliados; mientras que, de los hogares con jefes $66 \%$ fueron nucleares y $30 \%$ ampliados; cabe señalar que, en términos de condiciones de pobreza, las jefas sin pobreza conforman hogares nucleares y aquellas en pobreza hogares ampliados (48 y 55\%, respectivamente).

Por otra parte, la falta de educación sexual y la promoción de un modelo de género tradicional que plantea un comportamiento sexual activo en los hombres, ha promovido, entre otras situaciones, la fecundidad adolescente, la cual suele identificarse como una de las causas de la jefatura femenina del hogar. Así, es común que dichas mujeres jóvenes se incorporen como núcleo secundario a un hogar extendido/ ampliado que, a la vez, está encabezado por una mujer (Ariza y de Oliveira, 2007), originando que la mayoría de dichas madres solteras vivan con familiares y carezcan de vivienda propia (Lázaro y Martínez, 2003), lo que las hace aún más vulnerables.

Un indicador de lo anteriormente señalado es la dependencia demográfica. Se ha identificado que en los hogares encabezados por mujeres hay una mayor dependencia económica potencial, es decir, que más individuos en edad no laboral (menores de 15 y mayores de 65 años) dependen de ellas. Así, en 2018 la razón de dependencia demográfica en hogares con jefas fue de 61 personas potencialmente dependientes sobre 100 económicamente activas; en cuanto a los hogares con jefes, la relación es de 56/100. La situación se vuelve más dramática en hogares con jefas en pobreza, cuya relación es de 84/100.

Asociado a factores económicos, ante la falta de oportunidades y empleo, sobre todo en las comunidades rurales, los hombres emigran nacional o internacionalmente, de manera temporal o definitiva, olvidándose de sus responsabilidades o adquiriendo nuevas, al formar otras familias. Así, muchos hogares son abandonados, dejando la familia a cargo de mujeres viudas, divorciadas, madres solteras responsables de la producción y reproducción de los hogares (Lázaro et al., 2005).

Cuando las mujeres se enfrentan a la responsabilidad de mantener un hogar por sí solas, deben participar en el mercado laboral; sin embargo, existen numerosas desventajas en dicho terreno. Económicamente, ha habido un incremento de la participación femenina en el mercado laboral, lo que ha contribuido en el reconocimiento como cabezas de hogar; de 2008 a 2018 la tasa de participación económica creció 8 puntos (de 44 a 52\%).

Con base en la Organización Internacional del Trabajo (OIT, 2018), en América Latina la tasa de participación femenina es inferior que la masculina (50 y 74\%, respectivamente); igualmente, la tasa de desempleo para las mujeres es mayor y existe una brecha salarial importante, alrededor del $15 \%$ a nivel regional. Otro aspecto importante es que la situación laboral de las mujeres está frecuentemente asociada a situaciones 
irregulares o informales, tales como contratos temporales, actividades asociadas a labores domésticas y cuidado infantil, entre otras, lo cual promueve inseguridad social e inestabilidad laboral.

México es uno de los países de América Latina que presenta una de las brechas de género más grandes de la región: la segunda más alta (Kaplan y Piras, 2019). Así, el incremento de la tasa de participación femenina en el mercado laboral se ha visto acompañada de discriminación, la cual se ve reflejada en menores salarios, derechos laborales y prestigio social (Arceo-Gómez y Campos-Vázquez, 2014). Respecto al punto anterior, el trabajo doméstico remunerado es una de las actividades que las mujeres de escasos recursos realizan. Con base en datos de la Organización Internacional del Trabajo (OIT, 2016), $11.4 \%$ de las mujeres ocupadas en América Latina y el Caribe se encuentran en este sector y cerca del $80 \%$ lo hacen de manera informal. Las vulnerabilidades y carencias asociadas, ya de por sí, a este tipo de trabajo (bajas remuneraciones, falta de derechos laborales, inestabilidad laboral, no disponibilidad de seguridad social, falta de reconocimiento, entre otros), se suman a las impuestas por la pandemia Covid-19: incremento de medidas de higiene y limpieza, exposición al contagio por convivencia fuera de sus casas, limitaciones para acceder a sus lugares de trabajo, reducción de sus ingresos, por mencionar algunas situaciones.

El presente trabajo tiene por objetivo evidenciar, a partir de estadísticas descriptivas, el aumento en el número de hogares que cuentan con una jefatura femenina entre 2008 y 2020, así como examinar la evolución de algunos de los determinantes de ésta, es decir, se analiza empíricamente el impacto de las variables: nivel de educación formal con el cual cuenta el jefe del hogar, ingreso corriente trimestral del hogar y número de integrantes menores de 11 años en la probabilidad de que la jefatura del hogar esté a cargo de una mujer. Para realizar dicho análisis se emplean modelos probabilísticos de respuesta binaria (Logit y Probit) y datos de la Encuesta Nacional Ingreso Gasto de los Hogares para el periodo 2008-2020.

La hipótesis es que el número de hogares con jefatura del hogar femenina ha crecido en los últimos años debido a que ciertos factores (edad, educación, número de integrantes menores de edad y nivel de ingreso) inciden en la probabilidad de que dicha situación ocurra.

La presente investigación presenta evidencia empírica sobre el aumento en la participación de las mujeres en la jefatura de los hogares mexicanos, demostrando la importancia y necesidad de la ejecución de programas y proyectos que propicien mejoras en el tema de brechas de género existentes en el país, ya que, como mencionan algunos autores (Chant, 2003; Javed y Asif, 2019), la vulnerabilidad de caer en problemas de pobreza, y por ende, impactar de manera negativa en la calidad de vida de los integrantes de un hogar, aumenta cuando la jefatura de un hogar está a cargo de una mujer.

Es importante destacar que, como mencionan Muñoz et al. (2018), no se puede sugerir un vínculo directo entre la jefatura femenina, ya que los hogares encabezados por mujeres son un grupo muy heterogéneo y bien pueden reflejar autoselección, procesos demográficos, entre otros.

El artículo es de especial relevancia debido a que la literatura en torno al tema de la jefatura femenina del hogar y sus determinantes ha sido escasamente abordada, sobre todo de forma empírica, y aun en menor proporción para el caso mexicano. Igualmente, el fenómeno analizado adquiere una especial importancia en el contexto económico y sanitario actual, ante la crisis de la Covid-19.

El trabajo se encuentra estructurado de la siguiente manera: después de la introducción, se presenta la revisión de los trabajos realizados en torno al tema; posteriormente, se describen los datos y la metodología que se utilizaron para realizar el trabajo; a continuación, se presentan y analizan los resultados; y, por último, se concluye el trabajo. 


\section{Estado del arte}

El aumento en el número de mujeres que son responsables de sus hogares, alrededor del mundo, es un tema que aparece en la mayoría de los estudios especializados en el tema de la jefatura doméstica (Buvinic 1991; Wartenberg, 1999; Buvinic y Gupta 1997; Lloyd y Duffy 1995). En América Latina, las investigaciones sobre el incremento de los hogares encabezados por mujeres surgieron con fuerza en los años noventa y, al día de hoy, se verifica una importante producción sobre esta temática (Acosta Díaz, 2001).

Sesento García (2015) menciona que para hablar de las mujeres mexicanas se debe partir de la distinción entre aquellas que se encuentran en el contexto rural y las que se encuentran en el urbano. La primera categoría es considerada sin poder, dedicada a los deberes familiares y del hogar y en total subordinación ante el varón. Por otra parte, la mujer de ciudad o mujer urbana, en algunos casos tiene por objetivo principal sus estudios bajo el supuesto de tener una vida laboral en crecimiento en el país, razón por la cual soslaya su interés por encontrar una pareja estable, así como el de formar una familia y tener hijos.

Como señala Sesento García (2015), durante el siglo XXI la mujer mexicana dejó de ser en su totalidad rural y agrícola para trabajar en el sector industrial, propiciando de esta forma nuevas relaciones económicas y sociales, razón por la cual el tener hijos posee una menor importancia y si los tiene, entre menos sean mejor.

Rojas (2016) apunta que las relaciones familiares, parentales y conyugales han registrado cambios generacionales diferenciados si se consideran los estratos socioeconómicos y los grupos sociales, puesto que los cambios más significativos se presentan principalmente en los sectores sociales con mejores condiciones de vida, niveles educativo superiores y con residencia en ámbitos urbanos.

Son diversos los estudios que han evidenciado una tendencia de incremento de los hogares encabezados por mujeres en México, tanto a nivel nacional como regional (por ejemplo, en la frontera norte) (Acosta Díaz, 1995; Ariza y de Oliveira, 2001; García y Rojas, 2002; Navarro Ornelas y González Ramírez, 2010). A pesar de lo anterior, Vargas Valle y Navarro Ornelas (2013) señalan que la proporción de jefas de hogar podría estar subestimada, dado que la jefatura femenina que se logra apreciar corresponde a la autoidentificación o identificación de las mujeres, por parte de los miembros que integran el hogar, como responsables de los recursos domésticos; lo cual, en una cultura patriarcal como la mexicana, se asocia generalmente a la ausencia de una figura masculina dentro del hogar y no a la persona en quien recae mayoritariamente la manutención del mismo.

Acosta Díaz (2001) señala que debido a que no existen definiciones comunes o estandarizadas del concepto jefatura, su significado social es distinto, según sea el sexo de quien la posee; mientras que la jefatura masculina alude a un hogar con la pareja intacta y presente en el hogar, la jefatura femenina se asocia tradicionalmente al hogar de una mujer que no tiene una pareja masculina, ya sea por su estado civil: soltera, viuda, divorciada o separada. Para Di Marco (1998), como se menciona en Navarro Ornelas y González Ramírez (2010), el concepto de jefatura de hogar considera por lo menos dos situaciones: 1) establecer un orden jerárquico entre los miembros del hogar y 2) identificar al principal proveedor de recursos.

En este sentido, diversos autores utilizan diferentes significados respecto a los hogares con jefatura femenina. Para García (1998), son aquellos hogares en donde las mujeres son las principales responsables, en términos económicos, de sus familias. Por su parte, De Oliveira (1998) considera jefas de hogar tanto a las mujeres que no tienen cónyuge como a las que son jefas aun en presencia de éste, así como a las que encabezan el hogar por la ausencia temporal del varón, ya sea por cuestiones de migración interna o internacional. Chant (1999) se re- 
fiere a este tipo de hogares como las unidades domésticas encabezadas por mujeres solas, las cuales generalmente se encuentran separadas o son viudas.

Por otra parte, el papel de las mujeres dentro de las organizaciones, si bien ha presentado mejoras en los últimos años, éstas aún no son suficientes. Carrasco Macías (2004) señala que el uso del género como categoría para el análisis de la realidad social se omite al momento de exponer los procesos organizativos y el funcionamiento de las organizaciones. Aunado a lo anterior, la falta de atención a dicha categoría ha provocado la ignorancia y/o subestimación del papel de la mujer dentro de dichas instituciones.

Por su parte, Coronel Llamas et al. (2002) señalan que la teoría organizativa ha aceptado una ideología y valores esencialmente masculinos y que las cuestiones en torno al papel de las mujeres se han mantenido ausentes o han sido tratadas como irrelevantes, dentro del discurso sobre la organización.

Aunado y derivado de la subestimación del papel de las mujeres dentro de las organizaciones, existen barreras tanto externas como internas para las mujeres a puestos de gestión dentro de las organizaciones, siendo algunas de estas barreras autoimpuestas, al decidir libremente no promocionarse a puestos de mayor responsabilidad por el costo personal que ello supone, ya que consideran (de manera consciente o inconsciente) ser las responsables principales del cuidado de la familia y el hogar (Garcés, 2016), o bien por conflictos internos y presiones psicológicas que derivan en su autoexclusión de determinadas esferas del entorno laboral (Padilla Carmona, 2002), generando una falsa sensación de satisfacción laboral.

Sánchez Cañizares et al. (2007) realizaron un análisis de la satisfacción laboral desde la perspectiva de género con la aplicación de los modelos Probit y Logit; constataron que las mujeres presentan un nivel superior de satisfacción al de los hombres. Aguilar Barrera y Gutiérrez Pulido (2017) señalan que los cambios positivos en te- mas de transición demográfica, desarrollo humano y participación femenina en el mercado laboral se ven contrarrestados por situaciones como el embarazo adolescente, el aumento de los hogares monoparentales con una jefatura femenina y la prevalencia de la poca participación de los hombres en las actividades domésticas.

A su vez, Castillo et al. (2015) realizaron un estudio sobre la resiliencia en las familias monoparentales con jefatura femenina en contextos de pobreza en Colombia; señalan que las mujeres jefas de hogar bajo un contexto de pobreza y vulnerabilidad pueden construir la resiliencia en el ejercicio de su parentalidad. Otro estudio que relaciona la pobreza y la vulnerabilidad con las condiciones de vida material de los hogares monoparentales es el realizado por Mendoza Rivas y López Estrada (2013), el cual señala que, en comparación con el resto de los hogares en el estado de Nuevo León, no es posible sugerir un vínculo directo entre la pobreza y la vulnerabilidad, así como tampoco las situaciones de vida material de los hogares en estudio.

Romero-Picón y Chávez-Plazas (2013) realizaron una aproximación a la feminización de la pobreza y a la responsabilidad en familias desplazadas por la violencia; señalan que los bajos niveles de escolaridad, trabajos mal remunerados, problemas afectivos y la carga de responder solas por sus hogares son factores que agravan su situación de pobreza. Sosa Castro et al. (2019) realizaron un análisis cuantílico respecto a las brechas salariales desde una perspectiva de género en México; señalan que existe una brecha entre los ingresos de hogares con jefes de familia varones y los hogares con jefas de familia mujeres, siendo la variable que refiere al nivel educativo el factor que explica la diferencia en los ingresos.

Si bien son muchos los trabajos que abordan el tema de la jefatura femenina en los hogares, son pocos los que se refieren al tema de sus factores determinantes. Un ejemplo de esa literatura es el trabajo realizado por Nava Bolaños y Ham Chande (2014), que identifican y analizan los de- 
terminantes de la participación laboral de la población de 60 años o más en México mediante un modelo de probabilidad logística. Los resultados evidencian que la participación en el mercado laboral se explica mayormente por la jefatura del hogar.

\section{Metodología y datos}

\section{Datos}

En México no existe una encuesta especializada que permita analizar la trayectoria de las personas en términos de las decisiones que toman (encuesta panel); por ejemplo, tener hijos o vivir con una pareja. La mayoría de la información que se tiene es a partir de cortes transversales con representatividad estadística a nivel país y para subgrupos de la población (Ibarra López, 2019).

Dentro de este tipo de instrumentos destaca la Encuesta Nacional de Ingresos y Gastos en los Hogares (ENIGH), ${ }^{1}$ que realiza el Instituto Nacional de Estadística y Geografía (Inegi) de forma sistemática cada dos años en el país (Ibarra López, 2019) con el objetivo de brindar información concerniente, como su nombre lo indica, a la naturaleza de los ingresos y gastos en los hogares en términos de su monto, procedencia y distribución. Dichos datos son complementados con las características sociodemográficas de los integrantes del hogar; así como de la vivienda y de los recursos con los que cuenta la misma.

La principal razón por la cual se utiliza la ENIGH como fuente de información en el presente trabajo es que es una encuesta con una amplia diversidad de temas y se actualiza con mayor regularidad que un censo. Asimismo, mediante este instrumento se pueden identificar hogares monoparentales y conocer para todos ellos las características del jefe o jefa del hogar: las características socioeconómicas de la persona que administra o vive en un determinado hogar, el género, el número de hijos que tiene, su estado civil, entre otras.

1 A partir de 2008 se han realizado modificaciones en la ENIGH en términos de su precisión, atendiendo las recomendaciones de la Organización de las Naciones Unidas (ONU).
Para el desarrollo de este artículo se ocupan los datos publicados en la sección "microdatos" 2 , empleando las variables que refieren al sexo, ${ }^{3}$ edad $^{4}$ y nivel de educación formal ${ }^{5}$ del jefe del hogar, así como aquellas que describen el número de integrantes menores $^{6}$ de 11 años con los que cuenta, el ingreso corriente ${ }^{7}$ y el tipo de hogar. ${ }^{8}$ Las variables sexo, educación y hogar son variables de tipo nominal, mientras que las variables edad y menores son variables continuas. La variable sexo asume valores de 1 y 2 , si el jefe del hogar es varón o mujer, respectivamente. Por otra parte, el nivel educativo se establece dentro del rango ( $\mathrm{O}$ a $11)$, con base en lo siguiente:

01 Sin instrucción

02 Preescolar

O3 Primaria incompleta

O4 Primaria completa

O5 Secundaria incompleta

O6 Secundaria completa

07 Preparatoria incompleta

o8 Preparatoria completa

o9 Profesional incompleta

10 Profesional completa

11 Posgrado

2 Para el caso de la edición 2008, la encuesta fue realizada del 21 de agosto al 28 de noviembre de dicho año con una muestra de 35,146 viviendas, de las cuales se presentan los datos de 29,468 hogares: 7291 entrevistados tienen por jefe de familia a una mujer y 22,177 a un hombre. Asimismo, para 2020 la encuesta fue realizada del 21 de agosto al 28 de noviembre del mismo, con una muestra de 105,483 viviendas, de las cuales 25,776 de los hogares entrevistados tienen por jefe de familia a una mujer y 63,230 a un hombre, presentando de esta manera los datos de 89,006 hogares.

3 Distinción biológica que clasifica al jefe del hogar en hombre o mujer.

4 Años transcurridos entre la fecha de nacimiento del jefe del hogar y la fecha de la entrevista.

5 Nivel de instrucción del jefe del hogar.

6 Integrantes del hogar que tienen 11 o menos años de edad.

7 Suma de los ingresos por trabajo, los estímulos, alquiler y otros ingresos provenientes de rentas, de transferencias, de estimación del alquiler y de otros ingresos.

8 Diferenciación de los hogares a partir del tipo de relación consanguínea, legal, de afinidad o de costumbre entre el jefe (a) y los otros integrantes del hogar, sin considerar a los trabajadores domésticos ni a los familiares de éstos ni a los huéspedes. 
Asimismo, la variable hogar se encuentra dentro del rango ( 1 a 5 ) con base en lo siguiente:

\section{Unipersonal $^{9}$ \\ 2 Nuclear $^{10}$ \\ 3 Ampliado"11 \\ 4 Compuesto $^{12}$ \\ 5 Corresidente ${ }^{13}$}

\section{Metodología}

Dado que un Modelo de Probabilidad Lineal (MPL) supone que la probabilidad de respuesta es lineal en un conjunto de parámetros, $\beta_{j}$; y considerando que se busca la ocurrencia o no-ocurrencia de la participación de la mujer en la jefatura del hogar, el uso de un MPL presenta varios problemas, siendo el principal el acotamiento de la probabilidad entre 0 y 1 , y, en la mayoría de los casos, no es el más adecuado, razón por la cual se opta por el uso de un modelo de respuesta binaria de la forma:

$$
P(y=1 \mid x)=G\left(\beta_{0}+\beta_{1} x_{1}+\ldots+\beta_{K} x_{K}\right)=G\left(\beta_{0}+x \beta\right)
$$

donde $G$ es una función que toma valores estrictamente entre cero y uno: $0<$ $G(z)<1$, para todos números reales $(z)$ y $\chi \beta=\beta_{1} \chi_{1}+\ldots+\beta_{K} \chi_{K}$. Asegura de esta forma que las probabilidades de respuesta estimadas se encuentren únicamente entre cero y uno (Wooldridge, 2012).

Logit y Probit son modelos de respuesta binaria, en los cuales la variable dependiente es la probabilidad de respuesta. Ambos modelos utilizan la distribución normal de probabilidad acumulada, la cual se representa en la gráfica 1 y tiene una forma de $\mathrm{S}$ (función sigmoidea). Esta clase de modelos se caracteriza por poseer una distribución logística y normal estándar con media cero y varianza igual a 1 (Gujarati y Porter, 2010;

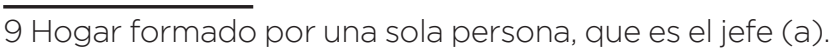
10 Hogar constituido por un solo grupo familiar primario.

11 Hogar formado por el jefe (a) y su grupo familiar primario más otros grupos familiares u otros parientes.

12 Hogar formado por un hogar nuclear o ampliado con personas sin parentesco con el jefe (a).

13 Hogar formado por dos o más personas que no tienen parentesco con el jefe (a).
Greene, 2012). Cabe mencionar que tanto las regresiones del modelo Logit como las del modelo Probit proporcionan probabilidades muy parecidas. La gráfica 1 muestra un ejemplo de probabilidades predichas por un modelo Logit y un modelo Probit.

\section{Gráfica 1 \\ Representación gráfica de las funciones logística y probabilística}

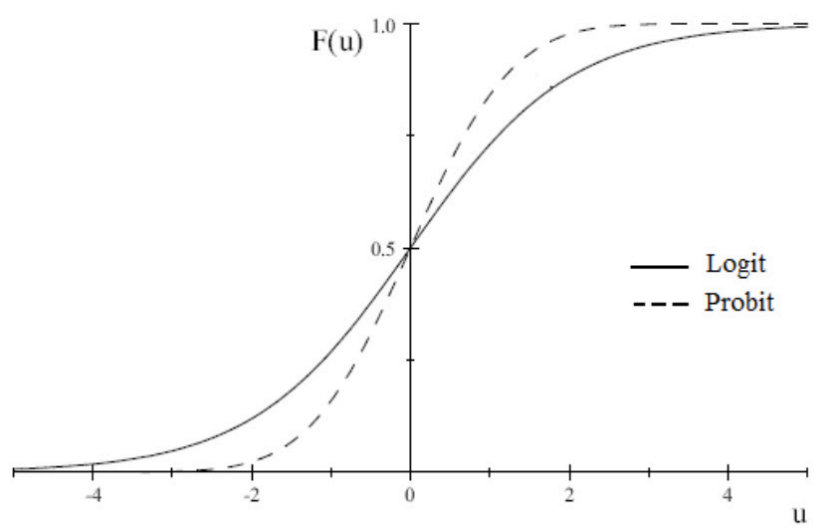

Fuente: Fonseca Nogueira Augusto (2010).

Dicho lo anterior, en el modelo Logit, G es la función logística:

$G(z)=\frac{e^{(z)}}{1+e^{(z)}}=\Lambda(z)$

que se encuentra entre cero y uno para todos los números reales z. Ésta es la función de distribución para una variable aleatoria logística estándar. Dentro del modelo Probit, $\mathrm{G}$ es la función de distribución acumulativa normal estándar (cdf), la cual es expresada como una integral (Corona Domínguez y Madrid Trillo, 2005):

$G(z)=\phi(z)=\int^{z} \phi(v) d v$ dar

donde $\phi(z)$ es la densidad normal están-

$$
\phi(z)=2 \pi^{-\frac{1}{2}} e^{\frac{-z^{2}}{2}}
$$

Escalante et al. (2013) mencionan que la especificación de la función permite definir distintos modelos, como es el caso de Probit y Logit (Baltagi et al. 2008), que asumen una distribución normal y logística, respec- 
tivamente. Para el caso del modelo Probit, la función se define como establece Greene (2012):

$\operatorname{Pr}\left(y_{i}=1 \mid x_{j}\right)=\int_{-\infty}^{\beta^{\prime} X} \varnothing(t) d t=\varnothing\left(\beta^{\prime} X\right)$

donde

$$
\varnothing(t)=\frac{1}{\sqrt{2 \pi}} e^{\left(\frac{-t^{2}}{2}\right)}
$$

Los modelos Logit y Probit son no lineales en los parámetros, por lo cual los estimadores se obtienen mediante el método de máxima verosimilitud. La función de verosimilitud logarítmica para el modelo Probit es:

$$
\ln L=\sum w_{j} \ln \varnothing\left(x_{j} b\right)+\sum w_{j}\left[1-\varnothing\left(x_{j} b\right)\right]
$$

El cálculo del cambio en la probabilidad se obtiene como la derivada parcial de la función de distribución, normal o logística (según sea el caso) respecto a la variable explicativa y ya que ésta es una función no lineal, el resultado va a cambiar en función de los valores de las variables explicativas (Greene, 2012).

Dicho lo anterior, y para los fines de este trabajo, se desarrolla un modelo de probabilidad para establecer relaciones entre un conjunto de variables explicativas que determinan la participación de la mujer en la jefatura del hogar:

$$
P(y=1 \mid x)=f\left(\beta_{0}+\beta_{1} x_{1}+\ldots+\beta_{K} x_{K}\right)
$$

donde $Y$ (variable dependiente) expresa la ocurrencia o no del evento y es de carácter dicotómica, la cual puede asumir los valores siguientes:

$$
Y=\left[\begin{array}{l}
1 \text { si el evento ocurre } \\
0 \text { si el evento no ocurre }
\end{array}\right]
$$

$\mathrm{P}=$ representa la probabilidad de que un individuo exhiba o desarrolle la característica de interés, en este caso que sea una mujer quien asume la jefatura del hogar. $\beta=$ parámetros o regresores del modelo, $x=$ variables independientes.
El modelo de probabilidad supone que las perturbaciones son homocedásticas y no correlacionadas, es decir, no adopta el supuesto de linealidad entre la variable dependiente (variable dicotómica) y las variables explicativas. Dentro del modelo a desarrollar se han incluido variables socioeconómicas tales como edad, educación, clase de hogar, ingreso. Los datos utilizados para elaborar la regresión corresponden a la ENIGH en su versión de 2008 a 2020.

Una vez determinadas las variables, el modelo tiene la siguiente especificación:

$P($ Sexo $=1)=f\left(\beta_{0}+\beta_{1}(\right.$ Edad $)+\beta_{2}($ Educacion $)+\beta_{3}($ Hogar $)+\beta_{4}($ Menores $)+\beta_{5}($ Ingreso $\left.)\right)(9)$

donde $P(\operatorname{Sexo}=1)$ es la probabilidad de ocurrencia de que la jefatura del hogar esté a cargo de una mujer, en función de las variables, edad, educación, hogar, menores e ingreso.

\section{Resultados y discusión}

\section{Análisis descriptivo}

Como se observa en la gráfica 2, la participación de las mujeres en la jefatura de los hogares mexicanos ha aumentado en los últimos 12 años alrededor de 4\%, ya que mientras ésta representaba $24.74 \%$ en 2008 , para 2020 la participación fue de $28.96 \%$ (cuadro 1). Esto se puede deber, entre otras situaciones, al aumento de la participación en el mercado laboral de las mujeres, los niveles de educación, las tasas de divorcio, la maternidad adolescente, la migración, el abandono, el escape de la violencia doméstica, etcétera.

\section{Cuadro 1 \\ Distribución de la jefatura de los hogares (2008-2020)}

\begin{tabular}{c|c|c|c|c}
\hline Jefe & Frecuencia & (\%) & Frecuencia & (\%) \\
\hline O (hombre) & 22,177 & 75.26 & 63,230 & 71.04 \\
\hline 1 (mujer) & 7,291 & 24.74 & 25,776 & 28.96 \\
\hline Total & 29,468 & 100 & 89,006 & 100 \\
\hline
\end{tabular}

Fuente: elaboración propia con base en datos de la muestra. 
Gráfica 2

Participación de las mujeres en la jefatura del hogar (\%)



Fuente: elaboración propia con base en datos de la ENIGH (2008-2020).

En 2008 la edad promedio de los jefes del hogar, sin distinción de sexo, fue de 48 años, mientras que para 2020 ésta aumentó a 51 años. Las mujeres jefas de hogar tenían en promedio 52 años, en tanto que la edad de los hombres en esta situación era de 47 años. Asimismo, para 2008 la mayoría de las mujeres jefas de hogar (22.8\%) se encontraba en un rango de edad entre 40 y 49 años, mientras que la mayoría de los hombres (13.1\%) en un rango entre $35 \mathrm{y}$ 39 años. Para 2020, la edad de la mayoría de las mujeres (10.9\%) se encontraba en un rango de entre 45 y 49 años, así como la de los hombres (12.10\%) (ver gráfica 3).

En la gráfica 3 se puede observar que, en ambos años, la participación de las mujeres en las jefaturas del hogar, en comparación con la de los hombres, aumenta después de los 55 años, lo cual es consistente con los resultados de Nava Bolaños y Ham Chande (2014).

Los resultados reflejan que la jefatura masculina del hogar para ambos años bajo análisis se centra en edades menores a 49 años, mientras que la jefatura femenina en edades de 35 años en adelante, lo cual podría deberse a que la esperanza de vida de mujeres es mayor que la de los hombres y las mujeres podrían quedar como jefas de hogar ante viudez; otra cuestión podría ser el abandono por parte del hombre y, finalmente, que la mujer haya escapado por temas de violencia en el hogar.
Los resultados de este análisis se pueden complementar con aquellos reportados por Coneval (2020), donde se señala que la incidencia de pobreza es mayor conforme las jefaturas son más jóvenes o de edad avanzada, indistintamente de su sexo. De 2008 a 2018, 40\% de jefaturas en el intervalo de 14 a 44 años, o de 65 años o más, presentó pobreza, en contraste con $30 \%$ de las jefaturas de 44 a 64 años.

\section{Gráfica 3}

Distribución de las edades de jefes y jefas del hogar por género (2008-2020)

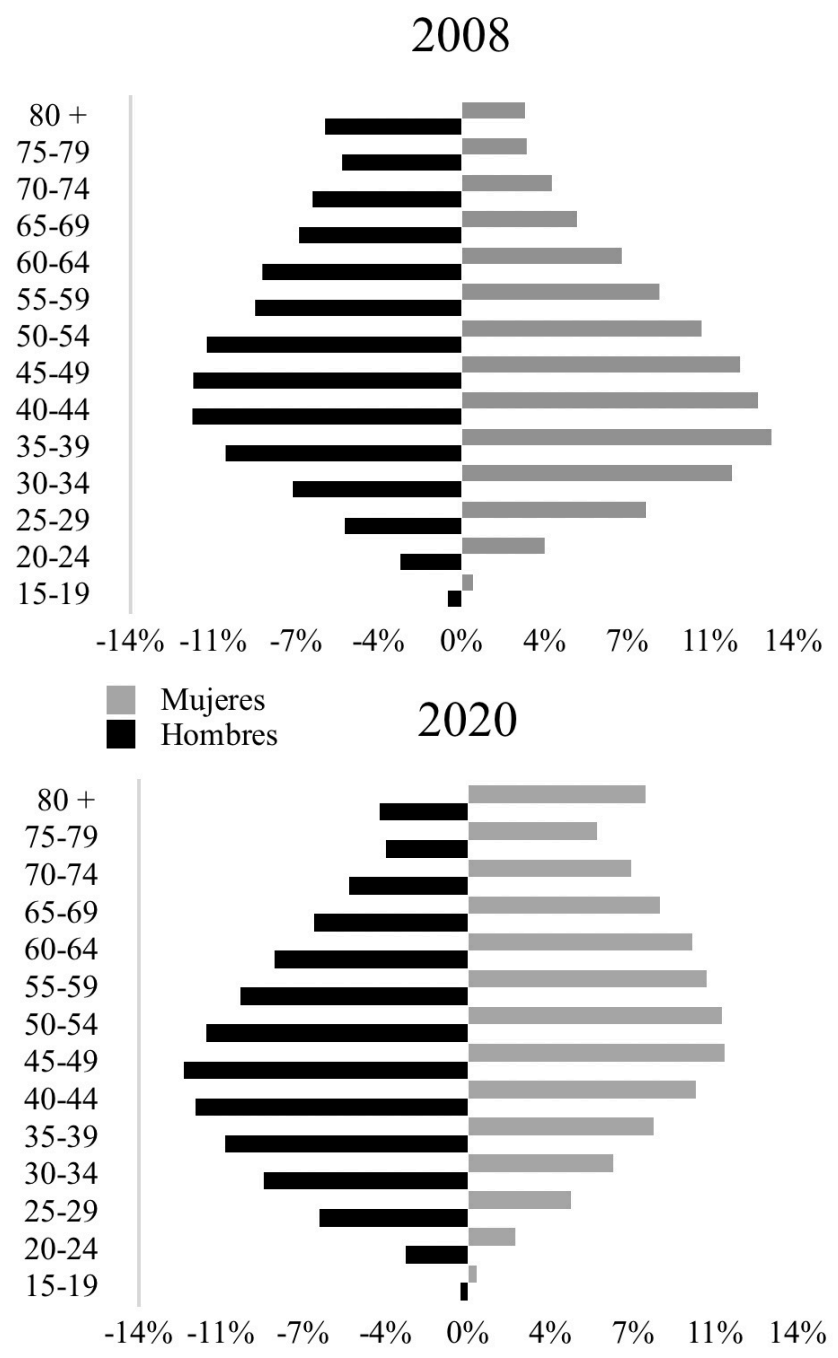

Fuente: elaboración propia con base en datos de la ENIGH (2008-2020). 
Con base en el Coneval (2020), de 2008 a 2018 el rezago educativo disminuyó para hombres y mujeres; así, la brecha entre ambos géneros en los grupos más jóvenes prácticamente es inexistente, mientras que en la población adulta se mantuvo. Durante el período citado, el rezago educativo para mujeres se redujo de 40 a $29 \%$ en mujeres y del 30 al $23 \%$ en hombres.

El nivel de educación formal promedio que reportaron los jefes del hogar para 2008 fue de secundaria incompleta (nivel 5). En 2020, este nivel fue el mismo en términos generales, pero en términos porcentuales la mayoría de los hombres y las mujeres reportaron un nivel de educación formal de secundaria completa (nivel 6) $-21.08 \%$ y 20.89 , respectivamente-, lo cual representa una mejora para las mujeres, ya que en 2008 la mayoría de ellas reportaba como nivel de educación formal primaria incompleta (nivel 3).

En la gráfica 4 se puede observar que, en ambos años, la proporción de hombres que tiene un nivel de educación formal de primaria completa (nivel 4) o inferior, es menor que la de las mujeres. Asimismo, se observa que en los niveles de educación profesional incompleta (nivel 9) y posgrado (nivel 11) la proporción entre hombres y mujeres jefes del hogar que cuentan con estos niveles de educación casi no ha variado en los últimos 12 años.

\section{Gráfica 4}

\section{Distribución del nivel de educación formal} del jefe y jefa del hogar

Fuente: elaboración propia con base en datos de la ENIGH (2008-2020). H-O8 y M-08 son los datos para hombres y mujeres en 2008, respectivamente, en tanto que $\mathrm{H}-20$ y $\mathrm{M}-20$ son los datos para hombres y mujeres en 2020.

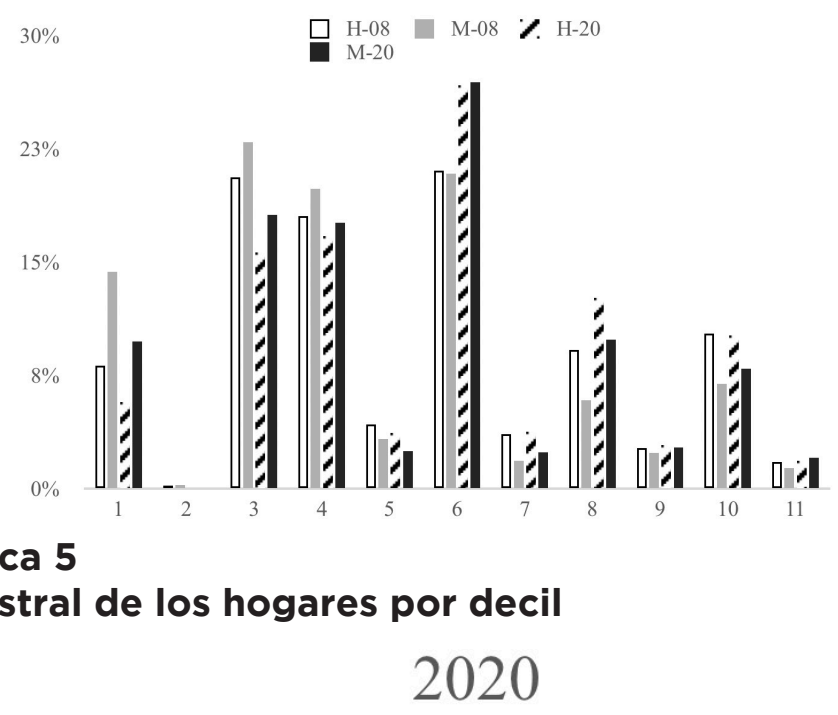

\section{Gráfica 5}

Distribución del ingreso trimestral de los hogares por decil

2008

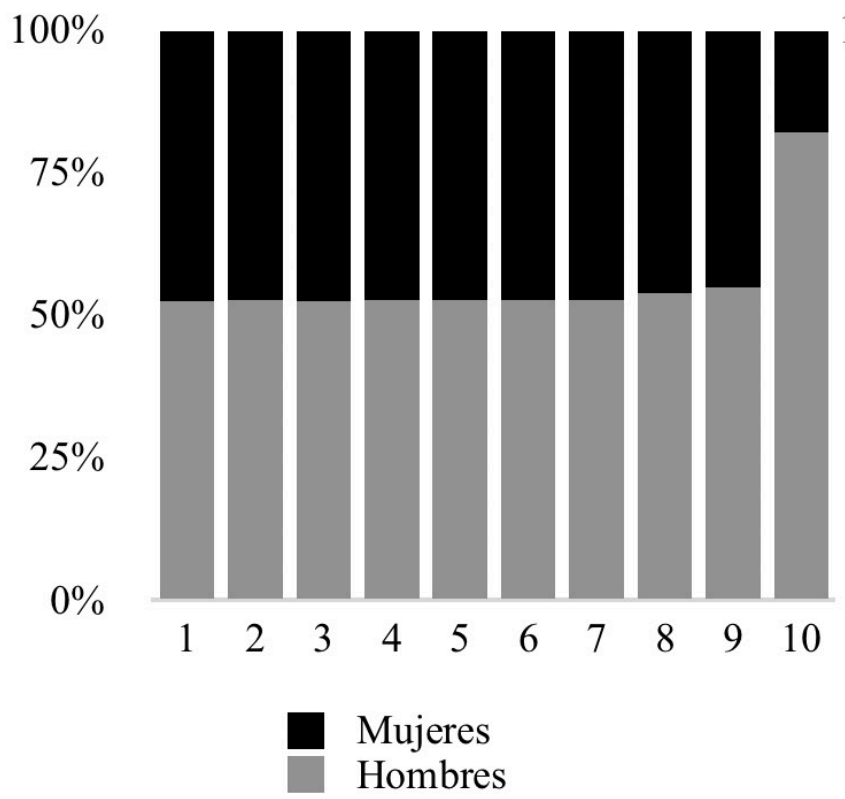

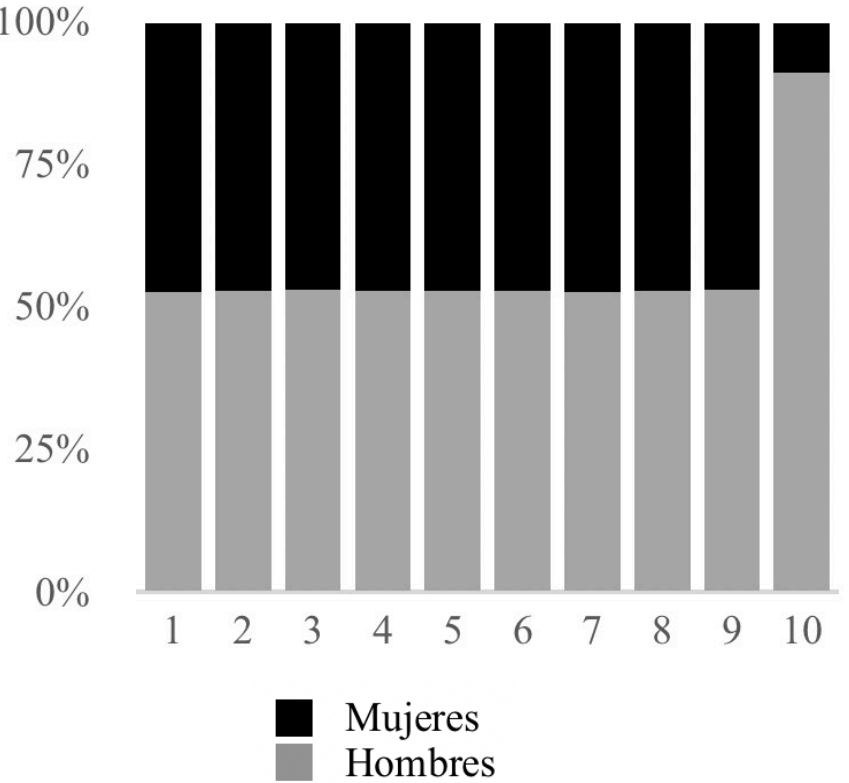

Fuente: elaboración propia con base en datos de la ENIGH (2008-2020). 
La distribución del ingreso corriente (trimestral) de los hogares por deciles se puede apreciar en la gráfica 5, en la cual se observa que, tanto en 2008 como en 2020, la mayor diferencia se presenta en el decil 10. Así, dentro del décimo decil, los hogares que cuentan con una mujer a cargo tienen un ingreso trimestral de 1,054,025 pesos, mientras que los hogares con hombres a cargo perciben 10,702,107 pesos; lo anterior es consistente con el fenómeno de techos de cristal, es decir, se podría explicar tal diferencia por la limitación en el ascenso laboral de las mujeres en algunas organizaciones.

Otro factor que explica la diferencia de resultados entre 2008 y 2020 es la pandemia propiciada por la Covid-19; la encuesta fue levantada de agosto a noviembre de dicho año. Así, muchas de las actividades laborales que han sido feminizadas están relacionadas con el cuidado de la salud, educación básica, asistencia en el hogar, cuidado infantil, entre otras. Así, aquellas jefas del hogar que estaban contratadas por tiempo determinado perdieron sus puestos de trabajo o redujeron su carga laboral, interrumpiendo o mermando sus ingresos.

En cuanto a los estratos socioeconómicos más bajos, las actividades laborales que desempeñan las jefas de familia pertenecientes a dicho nivel, generalmente se relacionan con actividades físicas (limpieza, maquila, cuidados, etcétera), por lo cual difícilmente se pueden realizar a distancia. Así, muchos de estos hogares carecieron de ingreso durante la pandemia o se vieron expuestos al contagio, soslayando el cuidado de su propia familia.

Al margen de la situación previamente señalada, el cierre de los centros educativos y de cuidado infantil ha impuesto una gran carga para las familias, ya que este grupo poblacional requiere de cuidados 24 horas al día, particularmente por parte de las mujeres, las cuales, con base en datos de la Comisión Económica para América Latina y el Caribe (CEPAL), destinan en promedio tres veces más tiempo al trabajo doméstico y de cuidado no remunerado que los hombres; previo a la crisis Covid-19 en la región de
América Latina y el Caribe, las mujeres destinaban entre 22 y 42 horas semanales a las actividades de cuidado y trabajo doméstico no remunerado (CEPAL, 2020).

Por otro lado, se puede apreciar que en ambos periodos (2008 y 2020) la diferencia de los ingresos en cada uno del resto de los deciles $\left(1^{\circ}\right.$ al $\left.9^{\circ}\right)$, no es tan amplia, ya que tanto los hogares que cuentan con la participación de una mujer como los que cuentan con la participación de un hombre en la jefatura del hogar cuentan con un ingreso casi similar.

Respecto al tipo de hogar, en ambos años la mayoría de estos eran de tipo nuclear y en promedio contaban con un integrante menor de 11 años (ver cuadro 2).

\section{Cuadro 2 Valores promedio}

\begin{tabular}{|l|r|r|}
\hline & $\mathbf{2 0 0 8}$ & $\mathbf{2 0 2 0}$ \\
\hline Edad & 48 & 51 \\
\hline Educación formal & 5 & 6 \\
\hline Menores & 1 & 1 \\
\hline Ingreso corriente & 37,464 & $47,838.47$ \\
\hline Clase de hogar & 2 & 2 \\
\hline
\end{tabular}

Fuente: elaboración propia con base en datos de la muestra.

\section{Geografía de la jefatura femenina del hogar}

En la gráfica 6 se observa la incidencia de la jefatura femenina por entidad, con el fin de complementar el análisis preliminar de los datos y examinar si hay algunos otros factores relacionados con dicha variable. Así, los estados con mayor incidencia en jefatura femenina son: Chihuahua, Baja California, Colima, Sinaloa y Querétaro.

Cabe señalar que tres de estos cinco se encuentran cerca de la frontera norte, por lo que se podría pensar que la dinámica migratoria incidiría en el desarrollo del fenómeno. No obstante, Zacatecas, Nayarit, Durango, Michoacán y Guanajuato fueron los estados que reportaron una mayor tasa de emigración durante 2020 (Inegi, 2020b). 


\section{Gráfica 6 \\ Análisis espacial de la incidencia de jefatura femenina por estado (2020)}

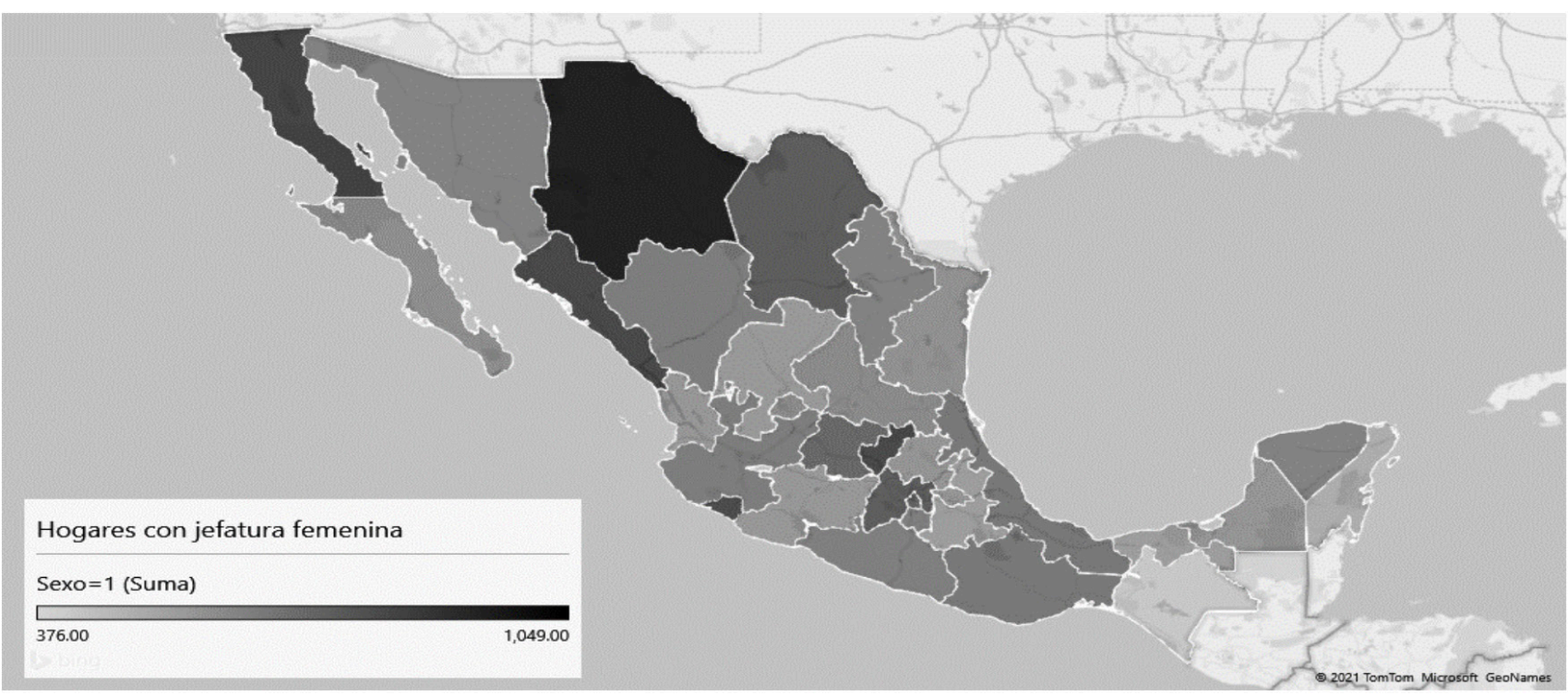

Fuente: elaboración propia con base en datos de la ENIGH (2020).

Algunas otras variables que, como se menciona en la introducción y revisión de la literatura, están relacionadas con la jefatura femenina son la violencia familiar. Durante 2020, los estados con mayor índice de violencia fueron el Estado de México, la Ciudad de México, Jalisco, Guanajuato, Baja California, Nuevo León, Chihuahua, Puebla y Veracruz (SESNSP, 2020). En este sentido, para Baja California y Chihuahua la incidencia de la jefatura femenina se puede relacionar con la violencia dentro del hogar.

Otra variable que también se asocia a la jefatura femenina es el embarazo en mujeres jóvenes entre 14 y 19 años, con base en la ENADID (Inegi, 2020b). Los estados que reportaron un mayor número fueron Tabasco, Tlaxcala, Chiapas, Puebla, Michoacán, Durango y Jalisco. Aparentemente, el embarazo juvenil podría estar asociada a otras variables, como la pobreza en el caso de Chiapas, Puebla, Michoacán y Jalisco (Coneval, 2020).

\section{Modelos Logit y Probit Pruebas de bondad de ajuste}

Existen varias pruebas de bondad de ajuste para comparar la diferencia entre valores predichos y valores observados. La prueba de bondad de ajuste de Pearson (P), mediante el estadístico Chi Cuadrado de Pearson, compara frecuencias observadas y esperadas en un escenario binomial. En dicha prueba, la hipótesis nula es que hay conformidad entre las frecuencias predichas y observadas a través de los patrones. Dicha hipótesis será rechazada para el nivel de significancia cuando el p-valor del contraste sea menor que el nivel fijado. Asimismo, la prueba de Hosmer-Lemeshow (HL) es otro método para medir la bondad de ajuste del modelo, consiste en comparar los valores observados con los valores pronosticados por el modelo. La hipótesis nula de esta prueba es que no hay diferencias entre los valores observados y los valores predichos. El rechazo de la hipótesis nula en cada una de las pruebas demostraría que el modelo no está bien ajustado (Iglesias Cabo, 2013).

Para el caso de los seis modelos utilizados en el presente trabajo las hipótesis nulas no son rechazadas, ya que el valor de la probabilidad del estadístico de prueba es mayor a 0.05 (nivel de significancia). Los resultados de las pruebas aplicadas a los modelos, en ambos periodos, se pueden observar dentro del cuadro 3. 


\section{Cuadro 3 \\ Resultados de las pruebas de bondad de ajuste}

\begin{tabular}{|c|c|c|c|c|c|c|c|c|c|c|c|c|c|c|}
\hline & \multicolumn{2}{|l|}{2008} & \multicolumn{2}{|l|}{2010} & \multicolumn{2}{|l|}{2012} & \multicolumn{2}{|l|}{2014} & \multicolumn{2}{|l|}{2016} & \multicolumn{2}{|l|}{2018} & \multicolumn{2}{|l|}{2020} \\
\hline \multicolumn{15}{|c|}{ Logit } \\
\hline P Chi2 & 29438.97 & $* * *$ & 27615.65 & $* * *$ & 8999.51 & $* * *$ & 19547.7 & $* * *$ & 101220.15 & & 74762.17 & $* * *$ & 89209.96 & *** \\
\hline HL Chi2 & 489.06 & $* *$ & 460.46 & $* * *$ & 460.46 & *** & 435.59 & $* * *$ & 1992.43 & ** & 9723.7 & $* *$ & 1772.2 & *** \\
\hline \multicolumn{15}{|c|}{ Probit } \\
\hline P Chi2 & $29,628.39$ & $* * *$ & $27,651.81$ & $* * *$ & 9005.54 & $* * *$ & $19,661.63$ & $* * *$ & $149,195.39$ & & $74,949.11$ & *** & $89,719.00$ & * \\
\hline HL Chi2 & 461.45 & $* * *$ & 453.85 & $* * *$ & 464.27 & $* * *$ & 471.5 & $* * *$ & 1994.65 & $*$ & 9073.95 & $* * *$ & 10227 & $* *$ \\
\hline
\end{tabular}

Nota: *, ${ }^{* *} \mathrm{y}^{* * *}$, denotan significancia al 10\%, 5\% y 1\%, respectivamente.

Fuente: elaboración propia con base en los resultados de las pruebas.

Una vez aplicadas las pruebas para determinar la bondad de ajuste de los modelos Pearson y Hosmer-Lemeshow, se observa dentro del cuadro 4 que en los modelos de 2008 a 2016 todas las variables utilizadas son estadísticamente significativas a un nivel de significancia del 99\%, mientras que en 2018 y 2020 la variable que hace referencia al nivel de educación formal que posee el jefe del hogar ya no es significativa. Lo anterior podría deberse a la disminución en la brecha de acceso a la educación entre hombres y mujeres, lo cual es consistente con lo señalado por Lechuga Montenegro et al. (2018).
Asimismo, se puede apreciar que con base en el signo de los coeficientes, las variables edad y hogar afectan de manera positiva a la probabilidad de que una mujer sea jefa del hogar; mientras que el nivel de educación formal, el número de habitantes del hogar menores de 11 años (menores) y el ingreso trimestral corriente (ingreso) lo hacen de manera negativa, es decir, la reducen.

Dado que los coeficientes de las variables en este tipo de modelos no se pueden interpretar de manera lineal, se hace uso de parámetros como la Razón de Odds o los efectos marginales, dependiendo del modelo seleccionado.

\section{Cuadro 4 \\ Coeficientes de los modelos Logit y Probit}

\begin{tabular}{|c|c|c|c|c|c|c|c|c|c|c|c|c|c|c|}
\hline Variable & \multicolumn{2}{|l|}{2008} & \multicolumn{2}{|l|}{2010} & \multicolumn{2}{|l|}{2012} & \multicolumn{2}{|l|}{2014} & \multicolumn{2}{|l|}{2016} & \multicolumn{2}{|l|}{2018} & \multicolumn{2}{|l|}{2020} \\
\hline \multicolumn{15}{|c|}{ Logit } \\
\hline & Coef. & 1 & Coef. & & Coef. & & Coef. & & Coef. & & Coef. & & Coef. & \\
\hline Edad2 & 0.0001129 & ${ }^{* * *}$ & 0.0001234 & ${ }^{* * *}$ & 0.0001313 & $* * *$ & 0.0001383 & $* * *$ & 0.0001328 & $* * *$ & 0.0001236 & *** & 0.0001262 & $* * *$ \\
\hline Educación & -0.0451866 & $* * *$ & -0.0456269 & ${ }^{* * *}$ & -0.0250712 & ${ }^{* *}$ & -0.0258853 & $* * *$ & -0.0154946 & $* * *$ & 0.0006451 & & -0.0041803 & \\
\hline Hogar & 0.2149538 & $* * *$ & 0.0918532 & ${ }^{* * *}$ & 0.1552253 & $* * *$ & 0.1797335 & $* * *$ & 0.1591034 & $* * *$ & 0.1635632 & $* * *$ & 0.1625671 & *** \\
\hline Ingreso & $-3.48 \mathrm{E}-06$ & ${ }^{* * *}$ & $-3.16 \mathrm{E}-06$ & ${ }^{* * *}$ & $-1.91 \mathrm{E}-06$ & $* *$ & $-3.94 \mathrm{E}-06$ & $* * *$ & $-3.12 \mathrm{E}-06$ & $* * *$ & $-3.62 \mathrm{E}-06$ & *** & $-3.69 \mathrm{E}-06$ & $* * *$ \\
\hline Menores & -0.2055576 & ${ }^{* * *}$ & -0.261967 & ${ }^{* * *}$ & -0.1942375 & $* * *$ & -0.1524718 & $* * *$ & -0.1603263 & $* * *$ & -0.1509941 & $* * *$ & -0.1407192 & *** \\
\hline _cons & -1.357706 & $* * *$ & -1.099753 & ${ }^{* * *}$ & -1.465179 & $* * *$ & -1.441465 & $* * *$ & -1.420755 & $* * *$ & -1.411973 & $* * *$ & -1.337621 & *** \\
\hline \multicolumn{15}{|c|}{ Probit } \\
\hline Edad2 & 0.0000681 & ${ }^{* * *}$ & 0.0000751 & ${ }^{* * *}$ & 0.0000787 & $* * *$ & 0.0000829 & $* * *$ & 0.0000787 & *** & 0.0000748 & *** & 0.0000764 & *** \\
\hline Educación & -0.0275216 & ${ }^{* * *}$ & -0.026837 & ${ }^{* * *}$ & -0.0150146 & ${ }^{* *}$ & -0.0157677 & $* * *$ & -0.0127508 & $* * *$ & -0.0001765 & & -0.0028302 & \\
\hline Hogar & 0.1279914 & *** & 0.0580571 & ${ }^{* * *}$ & 0.092839 & $* * *$ & 0.1063412 & $* * *$ & 0.0903237 & *** & 0.0979048 & $* * *$ & 0.0975386 & $* * *$ \\
\hline
\end{tabular}


Cuadro 4 (continuación)

\begin{tabular}{l|c|l|l|l|l|l|l|l|l|l|l|l|l|l}
\hline Ingreso & $-1.90 \mathrm{E}-06$ & $* * *$ & $-1.83 \mathrm{E}-06$ & $* * *$ & $-1.08 \mathrm{E}-06$ & $* *$ & $-2.14 \mathrm{E}-06$ & $* * *$ & $-1.24 \mathrm{E}-06$ & $* * *$ & $-2.00 \mathrm{E}-\mathrm{O} 6$ & $* * *$ & $-2.08 \mathrm{E}-06$ & $* * *$ \\
\hline Menores & -0.1194285 & $* * *$ & -0.149619 & $* * *$ & -0.1115906 & $* * *$ & -0.0895278 & $* * *$ & -0.0930573 & $* * *$ & -0.0891597 & $* * *$ & -0.083693 & $* * *$ \\
\hline _cons & -0.8318985 & $* * *$ & -0.6894597 & $* * *$ & -0.8954867 & $* * *$ & -0.8810987 & $* * *$ & -0.859859 & $* * *$ & -0.8670833 & $* * *$ & -0.8228617 & $* * *$ \\
\hline \hline
\end{tabular}

Nota: ${ }^{* *},{ }^{* * * *}$, denotan significancia al 10\%, 5\% y $1 \%$, respectivamente.

Fuente: elaboración propia.

Mediante la aplicación de los criterios de información Akaike (AIC) y Bayesiano (BIC), que sirven como parámetro para determinar qué modelo es mejor para la explicación de los datos, se observa que para 2008 y 2010 el mejor modelo es el Logit, mientras que de 2012 a 2020 es el Probit.

Una manera de interpretar los valores sobre el impacto de las variables en un modelo Logit es mediante el uso de la razón de Odds, la cual se obtiene al elevar el número de Euler (e) al valor de la beta estimada, que es el coeficiente de la variable, es decir $e^{\hat{\beta}_{j}}$, cuando este valor es mayor a uno se dice que la probabilidad de la ocurrencia del evento incrementa tantas veces como sea el valor; para el caso de los valores menores a uno, estos indican un decrecimiento en la probabilidad de la ocurrencia del evento. La forma de calcular cuántas veces decrecerá la probabilidad es estimar un cociente entre la unidad y el valor de razón de Odds $\left(\frac{1}{e^{\hat{\beta}_{j}}}\right)$.

\section{Cuadro 5 \\ Razón de Odds (2008-2010)}

\begin{tabular}{l|c|c|c|c}
\hline & \multicolumn{2}{|c|}{2008} & \multicolumn{2}{c}{2010} \\
Sexo & Odds Ratio & $\mathrm{P}>|\mathrm{z}|$ & Odds Ratio & $\mathrm{P}>|\mathrm{z}|$ \\
\hline Edad2 & 1.000113 & $* * *$ & 1.000123 & $* * *$ \\
\hline Educación & 0.9558191 & ${ }^{* * *}$ & 0.9553984 & $* * *$ \\
\hline Hogar & 1.239805 & ${ }^{* * *}$ & 1.096204 & $* * *$ \\
\hline Ingreso & 0.9999965 & $* * *$ & 0.9999968 & $* * *$ \\
\hline Menores & 0.8141932 & $* * *$ & 0.7695364 & $* * *$ \\
\hline _cons & 0.2572502 & $* * *$ & 0.3329533 & $* * *$ \\
\hline
\end{tabular}

Nota: *, ** $y^{* * *}$, denotan significancia al 10\%, 5\% y $1 \%$, respectivamente.

Fuente: Elaboración propia con base en los datos de la estimación.

En el cuadro 5 se puede observar que la probabilidad de que la jefatura de un hogar esté a cargo de una mujer en 2008 aumentaba 1.01 veces por cada año de edad adi- cional que poseyera el jefe del hogar, lo cual es consistente con los resultados de Nava Bolaños y Ham Chande (2014), y 1.24 veces dependiendo del tipo del hogar.

Por otra parte, la probabilidad de que la jefatura de un hogar en 2008 estuviera cargo de una mujer, decrecía 1.23 veces por cada integrante del hogar menor de 11 años y 1.05 veces por cada nivel adicional de educación.

Asimismo, se puede apreciar que, a lo largo del periodo de estudio, el impacto y la significancia de las variables se mantiene sin cambios significativos, siendo el cambio más significativo el que se presenta en 2010 para la variable menores, en términos de impacto y en 2012 para las variables educación y hogar, en términos de significancia (ver cuadro 5).

Dado que los valores calculados de los parámetros del modelo Probit no se relacionan de manera directa con la variable dependiente (Greene, 2012; Baltagi et al., 2008), para obtener una medida del impacto de cada variable en la probabilidad de que la jefatura de un hogar esté a cargo de una mujer, es necesario calcular el efecto marginal, es decir, el cambio parcial en la probabilidad ante un cambio marginal en la escala de una variable explicativa, dejando constante el resto de las variables. En el cuadro 6 se representan los efectos marginales de cada una de las variables del modelo. 


\section{Cuadro 6 \\ Efectos marginales del modelo Probit (2012-2020)}

\begin{tabular}{l|c|c|c|c|c|c|c|c|c|}
\hline & \multicolumn{3}{|c|}{2012} & \multicolumn{3}{|c|}{2014} & \multicolumn{2}{c}{2016} \\
\hline Variable & $\mathrm{dy} / \mathrm{dx}$ & $\mathrm{P}>|\mathrm{z}|$ & $\mathrm{X}$ & $\mathrm{dy} / \mathrm{dx}$ & $\mathrm{P}>|\mathrm{z}|$ & $\mathrm{X}$ & $\mathrm{dy} / \mathrm{dx}$ & $\mathrm{P}>|\mathrm{z}|$ & $\mathrm{X}$ \\
\hline Edad2 & 0.0000247 & $* * *$ & 2667.23 & 0.0000263 & $* * *$ & 2598.15 & 0.0000254 & $* * *$ & 2653.29 \\
\hline Educación & -0.004707 & $* *$ & 5.10176 & -0.004999 & $* * *$ & 5.50608 & -0.004107 & $* * *$ & 5.44855 \\
\hline Hogar & 0.0291028 & $* * *$ & 2.15408 & 0.0337141 & $* * *$ & 2.16592 & 0.0290944 & $* * *$ & 2.14833 \\
\hline Ingreso & $-3.37 \mathrm{E}-07$ & $* *$ & $33,675.30$ & $-6.78 \mathrm{E}-07$ & $* * *$ & 37262.8 & $-3.99 \mathrm{E}-07$ & $* * *$ & 42038.2 \\
\hline Menores & -0.034981 & $* * *$ & 0.860698 & -0.028384 & $* * *$ & 0.849428 & -0.029975 & $* * *$ & 0.812305 \\
\hline Edad2 & 0.0000247 & $* * *$ & 2736.69 & 0.0000257 & $* * *$ & 2866.5 & & & \\
\hline Educación & $-5.84 \mathrm{E}-05$ & & 5.52583 & $-8.51 \mathrm{E}-04$ & & 5.62028 & & \\
\hline Hogar & 0.0323653 & $* * *$ & 2.14792 & 0.0330741 & $* * *$ & 2.14918 & & \\
\hline Ingreso & $-6.62 \mathrm{E}-07$ & $* * *$ & $46,043.90$ & $-7.50 \mathrm{E}-07$ & $* * *$ & $47,838.50$ & & \\
\hline Menores & -0.029474 & $* * *$ & 0.760995 & -0.0286292 & $* * *$ & 0.689796 & & \\
\hline
\end{tabular}

Nota: *, ** $\mathrm{y}^{* * *}$, denotan significancia al 10\%, 5\% y 1\%, respectivamente.

Fuente: Elaboración propia con base en los datos de la estimación.

Los cambios más significativos en la probabilidad se registran en las variables tipo de hogar (3.3\%) y menores de 11 años (-2.9\%). Es decir, para 2020 la probabilidad de que la jefatura del hogar estuviera a cargo de una mujer aumentaba $3.3 \%$ dependiendo del tipo de hogar en el cual ésta fungía como jefa, lo cual es similar a lo expuesto por Dungumaro (2008), quien en su estudio encontró que las mujeres tienen más probabilidades de formar hogares extendidos que los hombres. Por otra parte, la probabilidad disminuía $2.9 \%$ por cada integrante menor de 11 años en el hogar. Los resultados sugieren que, a mayor edad es más probable que una mujer asuma la jefatura del hogar. Lo anterior se podría deber a separaciones, abandonos, divorcios o deceso del cónyuge. De manera contraria, la separación o abandono podría ser menos probable, entre mayor número de infantes (hijos) haya en la familia.

\section{Conclusiones}

La participación de las mujeres mexicanas en la jefatura de los hogares ha aumentado en los últimos 12 años y los factores que la determinan han variado en cuanto a su sig- nificancia e impacto. Tal es el caso del nivel de educación formal que posee el jefe del hogar, el cual dejó de ser un factor determinante de la jefatura femenina. Dentro de este estudio se demuestra el aumento en el número de hogares que cuentan con una mujer como jefa de familia en los últimos 12 años, lo cual apoya la hipótesis de este trabajo.

México se encuentra en el puesto número 50 , de un total de 149 , que conforman el índice global de brechas de género y es uno de los países de América Latina que presenta una de las brechas de genero más grandes de la región. Dentro de los principales problemas se encuentran: menores pago y derechos que se da hacia las mujeres por el mismo trabajo que a los hombres, menor ingreso en profesiones feminizadas, situaciones de inseguridad e informalidad laboral, por mencionar algunas situaciones desfavorables (Arceo-Gómez y Campos-Vázquez, 2014).

Por otra parte, y con base en los modelos probabilísticos Logit y Probit, se observa que las variables que impactan positivamente en la probabilidad de que una mujer sea la jefa del hogar son: edad y tipo de hogar; mientras que aquellas que impactan 
dicha probabilidad de manera negativa son: educación, ingreso y menores de 11 años en el hogar. Así, los hogares que tienen jefas de familia son aquellos en los que las mujeres tienen menor nivel educativo y por ende menor nivel de ingresos.

Los resultados evidencian el impacto y la persistencia de los roles tradicionales de género (las mujeres se ocupan en trabajos feminizados asociados a condiciones laborales precarias) y las brechas de género en el mercado laboral (menores salarios, prestaciones y prestigio social) (Kaplan y Piras, 2019).

Cabe señalar que otro hallazgo importante es que la variable educación formal con la cual cuenta el jefe del hogar perdió significancia a partir de 2018, lo cual podría referirse a mejoras en el acceso a la educación por parte de la población femenina.

Dicho lo anterior y con base en los resultados de esta investigación, el aumento en el número de hogares que cuentan con una mujer como jefa del hogar debe ser considerado como un llamado de atención a la sociedad y a los diferentes niveles de gobierno, a fin de que se promuevan programas y regulaciones que generen una efectiva reducción de las brechas de género. Como mencionan algunos autores (Buvinic, 1991; Wartenberg, 1999; Ariza y de Oliveira, 2007; Navarro Ornelas y González Ramírez, 2010; Romero-Picón y Chávez-Plazas, 2013), el riesgo de caer en pobreza puede aumentar cuando la jefatura de un hogar esté a cargo de una mujer.

El tema cobra especial relevancia en el contexto de la pandemia por la Covid-19, ya que los hogares cuya jefatura del hogar está a cargo de una mujer han sufrido los efectos de la agudización de las crisis económica, sanitaria y de los cuidados. Tal es el caso del Estado de México, que para 2020 era una de las entidades que presenta una mayor participación de la mujer en la jefatura del hogar (Inegi, 2020b) y ha sido uno de los estados que acusa una mayor tasa de casos positivos de Covid-19 durante 2020, aumentando de esta manera el tiempo dedicado al cuidado de los enfermos, ya que la tasa de pacientes ambulatorios es superior al 75\%, así como el cuidado de los integrantes del hogar (Conacyt, 2020).

Las futuras líneas de investigación podrían estar relacionadas con el análisis de otras características asociadas a los hogares cuya jefatura esté a cargo de una mujer. Igualmente, se podría comparar la situación a nivel América Latina.

\section{Fuentes consultadas}

Acosta Díaz, Félix (2001), "Jefatura de hogar femenina y bienestar familiar: resultados de la investigación empírica", Papeles de Población, 7 (28), Toluca, Universidad Autónoma del Estado de México, pp. 41-97, <https://bit.ly/3Bj$29 \mathrm{fH}>, 21$ de enero de 2021.

Acosta Díaz, Félix (1995), "Participación femenina, estrategias familiares de vida y jefatura femenina de hogar: Ios problemas de la jefatura declarada", Estudios Demográficos y Urbanos, 10 (3), Ciudad de México, El Colegio de México, A.C., pp. 545-568, doi: http://dx.doi. org/10.24201/edu.v10i3.954

Aguilar Barrera, Martha Elena y Gutiérrez Pulido, Humberto (2017), "Desigualdad de género y cambios sociodemográficos en México", Nóesis: Revista de Ciencias Sociales y Humanidades, 26 (51), Ciudad Juárez, Universidad Autónoma de Ciudad Juárez, pp. 2-19, doi: https:// doi.org/10.20983/noesis.2017.1.1

Arceo-Gómez, Eva O. y Campos-Vázquez, Raymundo M. (2014), "Evolución de la brecha salarial de género en México", El trimestre económico, 81 (323), Ciudad de México, Fondo de Cultura Económica, pp. 619-653.

Ariza, Marina y De Oliveira, Orlandina (2007), "Familias, pobreza y desigualdad social en Latinoamérica: una mirada comparativa", Estudios demográficos y urbanos, 22 (1), Ciudad de México, El Colegio de México, A.C., pp. 9-42, doi: https:// dx.doi.org/10.24201/edu.v22i1.1292 
Ariza, Marina y De Oliveira, Orlandina (2001), "Familias en transición y marcos conceptuales en redefinición”, Papeles de Población, 7 (28), Toluca, Universidad Autónoma del Estado de México, pp. 9-39, <https://bit.ly/2ZjSys2>, 21 de enero de 2021.

Baltagi, Badi H., Demetriades, Panicos y Law, Siong Hook (2008), "Financial development and openness: Evidence from panel data", Journal of development economics, 89 (2), Rhode Island, Brown University, pp. 285296, doi: https://doi.org/10.1016/j.jdeveco.2008.06.006

Buvinic, M. (1991), "La vulnerabilidad de los hogares con jefatura femenina: preguntas y opciones de política para América latina y el Caribe", Serie mujer y desarrollo, núm. 8, LC/L.611, Santiago de Chile, CEPAL/Unidad Mujer y Desarrollo, <https://bit.ly/312pndO>, 21 de enero de 2021.

Buvinic, Mayra y Gupta, Geeta (1997), "Female-Headed Households and Female-Maintained Families: Are They Worth Targeting to Reduce Poverty in Developing Countries?", Economic Development and Cultural Change, 45 (2), Chicago, The University of Chicago Press, pp. 259-280, doi: http://dx.doi. org/10.1086/452273

Carrasco Macías, María José (2004), "Participación y poder de la mujer en las organizaciones educativas", Revista de Educación, (6), Huelva, Universidad de Huelva, pp. 75-87, <https://bit.ly/ 3GkxFOm>, 21 de enero de 2021.

Castillo, Jennifer; Galarza, Dulce M. y González, Rocío A. (2015), "Resiliencia en familias monoparentales con jefatura femenina en contextos de pobreza", Revista Guillermo De Ockham, 13 (2), Cali, Universidad de San Buenaventura, pp. 45-54, doi: https://doi. org/10.21500/22563202.2063

CEPAL (Comisión Económica para América Latina y el Caribe) (2020), "La pandemia del COVID-19 profundiza la crisis de los cuidados en América Latina y el
Caribe", <https://bit.ly/3jBEW2o>, 21 de enero de 2021.

Chant, Sylvia (2003), "Female household headship and the feminization of poverty: facts, fictions and forward strategies", New Working Paper Series (9), Londres, London School of Economics and Political Science, <https://bit. ly/3md5etC>, 22 de enero de 2021.

Chant, Sylvia (1999), "Las unidades domésticas encabezadas por mujeres en México y Costa Rica: perspectivas populares y globales sobre las madres sin pareja", en Mercedes González de la Rocha (coord.), Divergencias del modelo tradicional: Hogares de jefatura femenina en América Latina, Ciudad de México, Ciesas/Plaza y Valdés.

Conacyt (Consejo Nacional de Ciencia y Tecnología) (2020), "COVID-19. Tablero México", Ciudad de México, Conacyt, <https://bit.ly/3GilS30>, 24 de enero de 2021.

Coneval (Consejo Nacional de Evaluación de la Política de Desarrollo Social) (2020), "Pobreza y género en México: hacia un sistema de indicadores, información 2008-2018", Ciudad de México, Coneval, <https://bit.ly/3nmmNqw>, 23 de enero de 2021.

Corona Domínguez, Michele Alexandra y Madrid Trillo, María Fernanda (2005), "Análisis de las determinantes del desempleo y su duración 1989-2001”, tesis de licenciatura, Universidad de las Américas, Cholula, <https://bit. ly/3nsCtsh>, 25 de enero de 2021.

Coronel Llamas, José Manuel; Moreno Sánchez, Emilia y Padilla-Carmona, Teresa (2002), "La gestión y el liderazgo como procesos organizativos: contribuciones y retos planteados desde una óptica de género", Revista de Educación, 327, Huelva, Universidad de Huelva, pp. 157-168, <https://bit.ly/3jBIxNW>, 24 de enero de 2021.

De Oliveira, Orlandina (1998), "Familia y relaciones de género en México", en Bea- 
triz Schmukler (coord.), Familias y relaciones de género en transformación, Ciudad de México, Population Council/ Edamex, pp. 23-52.

Di Marco, Graciela (1998), "La jefatura de hogar, ¿feminización de la pobreza?", en Beatriz Schmukler (coord.), Familias y relaciones de género en transformación, Ciudad de México, Population Council/Edamex, pp. 209-251.

Dungumaro, Esther W. (2008), "Gender Differentials in Household Structure and Socioeconomic Characteristics in South Africa", Journal of Comparative Family Studies, 39 (4), Toronto, Universidad de Toronto, pp. 429-451, <https:// bit.Iy/3vIDY9v>, 26 de enero de 2021.

Escalante, Roberto; Catalán, Horacio y Basurto, Saúl (2013), "Determinantes del crédito en el sector agropecuario mexicano: un análisis mediante un modelo Probit", Cuadernos De Desarrollo Rural, 10 (71), Bogotá, Pontificia Universidad Javeriana, pp. 101-124, doi: https:// doi.org/10.11144/Javeriana.cdr10-71.dcsa

Fonseca Nogueira Augusto, Margarida Soudo (2010), "The Indirect Costs of Diabetes in Portugal", tesis de maestría, Universidade Católica Portuguesa, Lisboa.

Garcés, Eva (2016), "Mujer y carrera profesional. ¿Techo de cristal o techo de cemento?", W360 Congress, <https://bit. ly/30uPjb3>, 21 de enero de 2021.

García, Brígida (1998), "Dinámica familiar, pobreza y calidad de vida: una perspectiva mexicana y latinoamericana", en Beatriz Schmukler (coord.), Familias y relaciones de género en transformación, Ciudad de México, Population Council/Edamex, pp. 53-79.

García, Brígida y Rojas, Olga (2002), "Los hogares latinoamericanos durante la segunda mitad del siglo XX. Una perspectiva sociodemográfica”, Estudios Demográficos y Urbanos, 17 (2), Ciudad de México, El Colegio de México, pp. 261-288, doi: http://dx.doi. org/10.24201/edu.v17i2.1139
Greene, William H. (2012), Análisis econométrico, Nueva Jersey, Prentice Hall.

Gujarati, Damodar N. y Porter, Dawn C. (2010), Econometría, Ciudad de México, McGraw Hill.

Ibarra López, Ignacio (2019), "La conformación de hogares con hijos en México: el papel del ingreso, la edad y la desigualdad salarial", Estudios demográficos y urbanos, 34 (3), Ciudad de México, El Colegio de México, pp. 535-567.

Iglesias Cabo, Tania (2013), "Métodos de Bondad de Ajuste en Regresión Logística", tesis de maestría, Universidad de Granada, Granada.

Inegi (Instituto Nacional de Geografía y Estadística) (2020a), "Encuesta Nacional de Ingresos y Gastos de los Hogares (ENIGH). 2020 Nueva Construcción", Ciudad de México, Inegi, <https://bit. ly/3b77X1v>, 26 de enero de 2021.

Inegi (Instituto Nacional de Geografía y Estadística) (2020b), "Encuesta Nacional de la Dinámica Demográfica. Microdatos", Ciudad de México, Instituto Nacional de Geografía y Estadística, <https://bit.ly/3mdwtUP>, 26 de enero de 2021.

Inegi (Instituto Nacional de Geografía y Estadística) (2018), "Encuesta Nacional de Ingresos y Gastos de los Hogares (ENIGH). 2018 Nueva Construcción", Ciudad de México, Inegi, <https://bit. ly/3EgSfgQ>, 26 de enero de 2021.

Inegi (Instituto Nacional de Geografía y Estadística) (2016), "Encuesta Nacional de Ingresos y Gastos de los Hogares (ENIGH). 2016 Nueva Construcción", Ciudad de México, Inegi, <https://bit. ly/3jDti7b>, 26 de enero de 2021.

Inegi (Instituto Nacional de Geografía y Estadística) (2014), "Encuesta Nacional de Ingresos y Gastos de los Hogares (ENIGH). 2014 Nueva Construcción", Ciudad de México, Inegi, <https://bit. Iy/3jx8Y7u>, 26 de enero de 2021. 
Inegi (Instituto Nacional de Geografía y Estadística) (2012), "Encuesta Nacional de Ingresos y Gastos de los Hogares (ENIGH). 2012 Nueva Construcción", Ciudad de México, Inegi, <https://bit. ly/3B942Md>, 26 de enero de 2021.

Inegi (Instituto Nacional de Geografía y Estadística) (2010), "Encuesta Nacional de Ingresos y Gastos de los Hogares (ENIGH). 2010 Nueva Construcción", Ciudad de México, Inegi, <https://bit. ly/3m|9NSV>, 26 de enero de 2021.

Inegi (Instituto Nacional de Geografía y Estadística) (2008), "Encuesta Nacional de Ingresos y Gastos de los Hogares (ENIGH). 2008 Nueva Construcción", Ciudad de México, Inegi, <https://bit. ly/3nwXRwJ>, 26 de enero de 2021.

Javed, Zahoor Hussain y Asif, Auesha (2019), "Female households and poverty: A case study of Faisalabad District", International Journal of peace and development studies, 2 (2), Faisalabad, University Faisalabad, pp. 37-44, <https:// bit.ly/2ZtHOmc>, 15 de enero de 2021.

Kaplan, David y Piras, Claudia (2019), "Brechas de género en el mercado laboral mexicano: comparaciones internacionales y recomendaciones de política pública", Revista de Economía Mexicana (4), Ciudad de México, Universidad Nacional Autónoma de México, pp. 138-165.

Lázaro, Rosa y Martínez, Beatriz (2003), "Mujeres jefas de Hogar y Relaciones de Género en los Municipios de Doctor Mora y Victoria, Guanajuato", Espacios Múltiples. Horas Interminables: Quehaceres de mujeres, Guanajuato, Instituto de las Mujeres Guanajuatenses, pp. 23-86.

Lázaro, Rosa; Zapata Martelo, Emma; Martínez, Beatriz y Alberti, Pilar (2005), "Jefatura femenina de hogar y transformaciones en los modelos de género tradicionales en dos municipios de Guanajuato", La ventana. Revista de estudios de género, 3 (22), Guadalajara, Universidad de Guadalajara, pp. 219-268.
Lechuga Montenegro, Jesús; Ramírez Argumosa, Giovanna y Guerrero Tostado, Maricruz (2018), "Educación y género. El largo trayecto de la mujer hacia la modernidad en México", Economía UNAM, 15 (43), Ciudad de México, Universidad Nacional Autónoma de México, pp. 110-139.

Lloyd, Cynthia B. y Duffy, Niev (1995), "Families in Transition", en Judith Bruce, Cynthia. B. Lloyd y Ann Leonard, Families in Focus. New Perspectives in mothers, fathers and children, Nueva York, The Population Council, pp. 5-24.

Mendoza Rivas, Luis Alberto y López Estrada, Raúl Eduardo (2013), "Los hogares monoparentales con jefatura femenina en Nuevo León", Prospectiva. Revista de Trabajo Social e Intervención Social, (18) Cali, Universidad del Valle, pp. 383410, doi: https://doi.org/10.25100/prts. v0i18.1136

Muñoz Boudet, Ana María; Buitrago, Paola; Leroy De La Briere, Benedicte; Newhouse, David. L.; Rubiano Matulevich, Eeliana C.; Scott, Kinnon y Suarez-Becerra, Pablo (2018), "Gender differences in poverty and Household Composition through the Life-cycle: A global perspective", Policy Research Working Paper núm. 8360, Washington, Banco Mundial, <https://bit.ly/3mdy4dp>, 10 de enero de 2021.

Nava Bolaños, Isalia y Ham Chande, Roberto (2014), "Determinantes de la participación laboral de la población de 60 años o más en México", Papeles de población, 20 (81), Toluca, Universidad Autónoma del Estado de México, pp. 59-87.

Navarro Ornelas, Ana María y González Ramírez, Raúl Sergio (2010), "Los hogares con jefatura femenina y la calidad de vida. Chihuahua y Tijuana, 2005", Estudios demográficos y urbanos, 25 (1), Ciudad de México, El Colegio de México, pp. 45-72, doi: http://dx.doi. org/10.24201/edu.v25i1.1367

OIT (Organización Internacional del Trabajo) (2016), Políticas de formalización 
del trabajo doméstico remunerado en América Latina y El Caribe, Lima, OlT-Programa de Promoción de la Formalización en América Latina y el Caribe (FORLAC), <https://bit.ly/3nvqtWX>, 28 de enero de 2021.

OIT (Organización Internacional del Trabajo) (2018), "OIT: La participación laboral de las mujeres aumenta, pero el camino a la igualdad aún es largo en América Latina y el Caribe", comunicado de prensa, 8 de marzo de 2018, Lima, OIT, <https://bit.ly/3jC6EvY>, 28 de enero de 2021.

Padilla Carmona, María Teresa (2002), "Desarrollo profesional femenino: la identidad de la mujer en el mundo laboral", en Emilia Moreno Sánchez y Sonia Villegas López (eds.), Introducción a los estudios de la mujer: una mirada desde las Ciencias Sociales, Huelva, Diputación provincial de Huelva, pp. 73-93.

Rojas, Olga Lorena (2016), "Mujeres, hombres y vida familiar en México. Persistencia de la inequidad de género anclada en la desigualdad social", Revista Interdisciplinaria de Estudios de Género de El Colegio de México, 2 (3), Ciudad de México, El Colegio de México, pp. 73-101, doi: https://doi.org/10.24201/ eg.v2i3.4

Romero-Picón, Yuri y Chávez-Plazas, Yuri (2013), "Jefaturas femeninas: una aproximación a la feminización de la pobreza y de la responsabilidad en familias desplazadas por la violencia", Tabula Rasa, (18), Bogotá, Universidad Colegio Mayor de Cundinamarca, pp. 277-286, <https://bit.ly/3Ck3bJl>, 30 de enero de 2021.

Sánchez Cañizares, Sandra M.; Fuentes García, Fernando J. y Artacho Ruiz, Carlos (2007), "La perspectiva de género en el análisis de la satisfacción laboral: una aplicación empírica mediante modelos logit y probit", Cuadernos de gestión, 7 (2), Bilbao, Universidad del País Vasco, pp. 55-67, <https://bit.ly/3EjDbyM>, 31 de enero de 2021.
Sesento García, Leticia (2015), "El papel de la mujer en el desarrollo de México", Revista Contribuciones a las Ciencias Sociales, núm. 27, Morelia, Universidad Michoacana de San Nicolás de Hidalgo, <https://bit.ly/3Cw2Cgl>, 30 de enero de 2021.

SESNSP (Secretariado Ejecutivo del Sistema Nacional de Seguridad Pública) (2020), "Datos Abiertos de Incidencia Delictiva", Ciudad de México, Gobierno de México, <https://bit.ly/3BsFi1H>, 2 de febrero de 2021.

Sosa Castro, Miriam Magnolia, Martínez-Preece, Marissa y Zubieta Badillo, Christian (2019), "Brecha de los ingresos en México desde una perspectiva de género", en Elvia Espinosa Infante (coord.), Mujeres en las organizaciones, Ciudad de México, Universidad Autónoma Metropolitana, pp. 159-178.

Vargas Valle, Eunice D. y Navarro Ornelas, Ana María (2013), "La estructura y la jefatura de los hogares de la frontera norte en la última década", Estudios fronterizos, 14 (27), Mexicali, Universidad Autónoma de Baja California, pp. 123-150, <https://bit.ly/3pEcj8x>, 5 de febrero de 2021.

Wartenberg, L. (1999), "Vulnerabilidad y jefatura en los hogares urbanos colombianos", en Mercedes González de la Rocha (comp), Divergencias del modelo tradicional: hogares de jefatura femenina en América Latina, Ciudad de México, Centro de Investigaciones y Estudios Superiores en Antropología Social/Plaza y Valdés, pp.77-96.

Wooldridge, Jeffrey M. (2012), Introductory Econometrics: A Modern Approach, Mason, Cengage.

Recibido: 26 de agosto de 2021. Aceptado: 5 de octubre de 2021. Publicado: 6 de enero de 2022. 


\section{Magnolia Miriam Sosa Castro}

Es doctora en Economía por la Universidad Nacional Autónoma de México, México. Actualmente es profesora-investigadora del Departamento de Economía, División de Ciencias Sociales y Humanidades, en la Universidad Autónoma Metropolitana, unidad Iztapalapa. Es miembro del Sistema Nacional de Investigadores, nivel I. Su línea de investigación actual es mercados financieros. Entre sus más recientes publicaciones destacan, como coautora: "Extreme volatility dependence in exchange rates", Cuadernos de Economía, 40 (82), Bogotá, Universidad Nacional de Colombia, pp. 25-55 (2021); "Financiarización y consumismo: multipolarismos y crisis Covid-19", Revista Mexicana de Ciencias Políticas y Sociales, 66 (242), Ciudad de México, Universidad Nacional Autónoma de México, pp. 345-372 (2021); "Impacto de la incertidumbre de la política económica y del tipo de cambio en el mercado accionario a nivel internacional (20012020): un análisis de causalidad y cointegración de panel", Paradigmas económicos. Revista de economía regional y sectorial, 13 (2), Toluca, Universidad Autónoma del Estado de México, pp. 55-78 (2021).

\section{Jorge Eduardo Castro Olivares}

Es maestro en Ciencias Administrativas por la Universidad Autónoma Metropolitana, México. Actualmente es estudiante del doctorado en Ciencias Administrativas en la Universidad Autónoma Metropolitana. Su línea de investigación actual es mercados financieros. Entre sus más recientes publicaciones se encuentran, como coautor: "Impacto de la incertidumbre de la política económica y del tipo de cambio en el mercado accionario a nivel internacional (20012020): un análisis de causalidad y cointegración de panel", Paradigmas económicos. Revista de economía regional y sectorial, 13 (2), Toluca, Universidad Autónoma del Estado de México, pp. 55-78 (2021); "Análisis de estrategias de inversión de diversificación internacional: portafolios tradicionales vs ETFs", Análisis económico, 34 (87), Ciudad de México, Universidad Autónoma Metropolitana, pp. 41-61 (2019); "Desigualdad Salarial en México. Un Enfoque Regional”, Revista Reporte, núm. 114, Ciudad de México, Cámara de Diputados, pp. 48-53 (2018). 


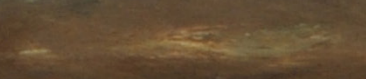

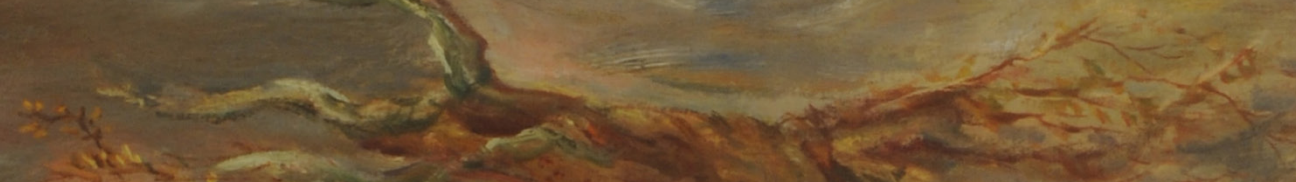

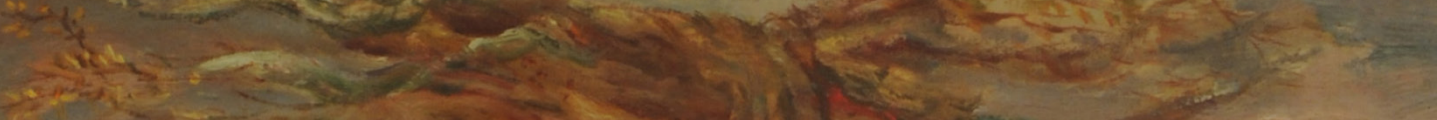

W.

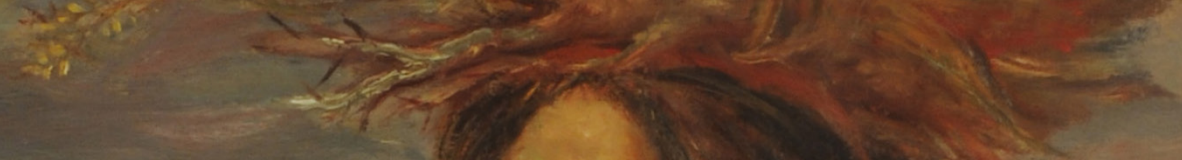

$\sqrt{3}+\frac{1}{4}$



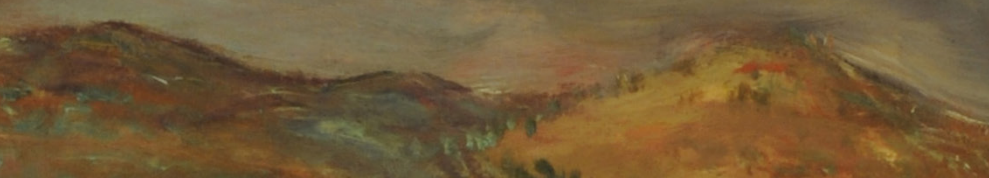

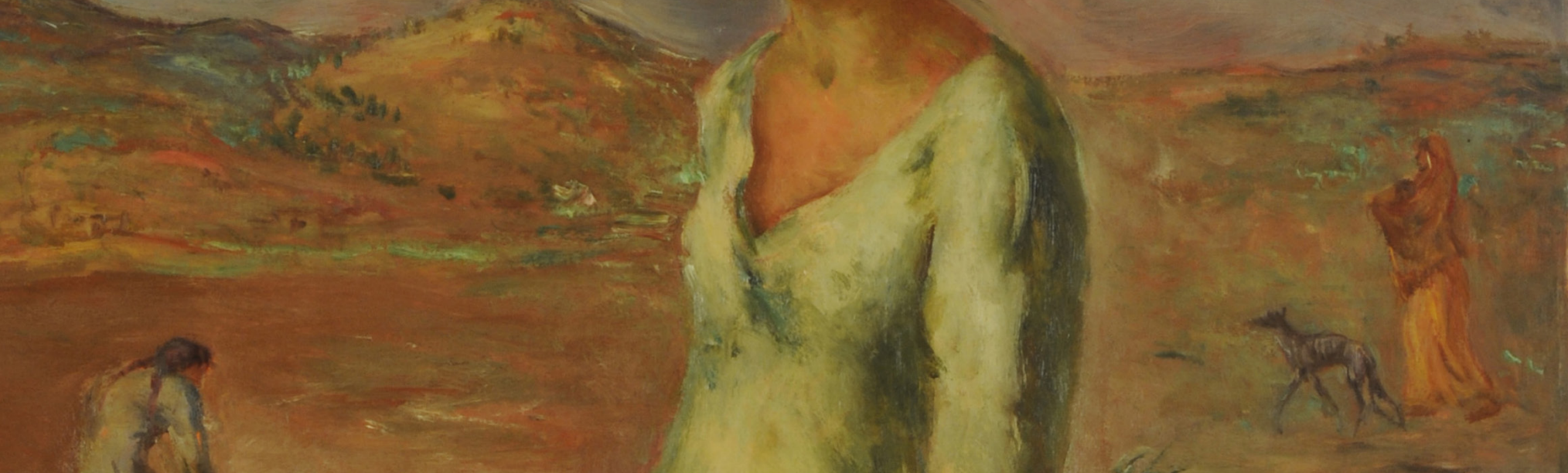

(2) 21

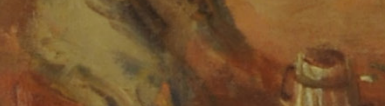



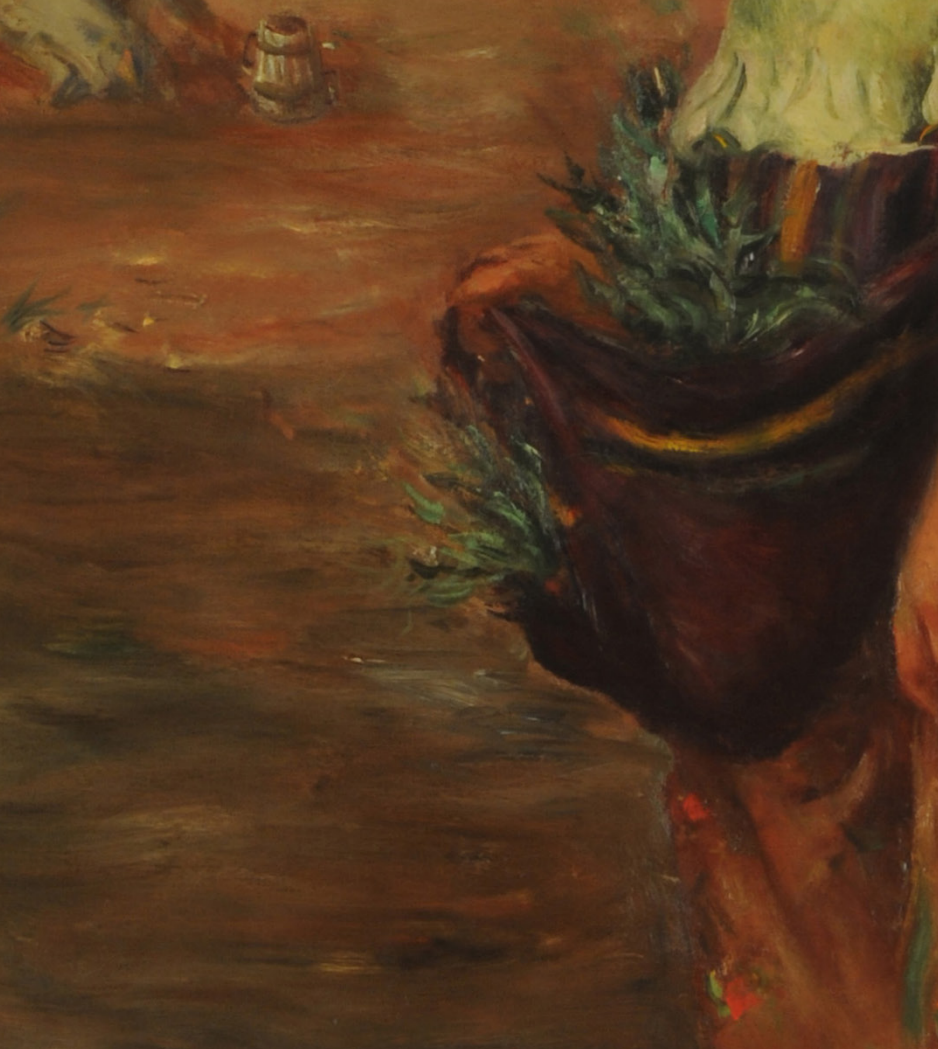

12
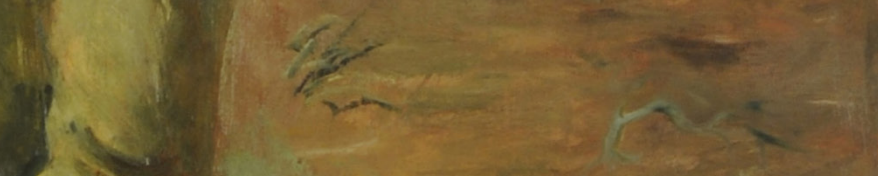

$\lim ^{-x^{x}} x^{2}$
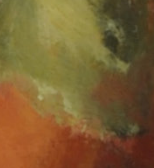
2.

3. $x^{2}=2 x$

(t) 